\title{
NOTAS
}

\section{ELEMENTOS DE ORALIDAD EN CARTAS DE SOLDADOS MEXICANOS DE LA ÉPOCA DE LA REVOLUCIÓN}

La comunicación por escrito presupone la familiaridad con la técnica cultural de representar los signos lingüísticos mediante formas gráficas. En español, éstas guardan una relación sistemática con las formas del lenguaje hablado porque las letras reproducen con cierta regularidad la secuencia de los fonemas. Sin embargo, las reglas de ortografía española comprenden elementos convencionales que marcan la diferencia entre una transcripción fonética y una escritura histórica. Aquellos aspectos convencionales hacen que la escritura española posea características propias que la independizan parcialmente de la lengua hablada y que tienen que ser aprendidas por los usuarios, además del principio fonético, básico del sistema de escritura del español ${ }^{1}$. Pero aparte de estas diferencias entre la lengua hablada y la lengua escrita, que conciernen a la transición de un medio auditivo a uno gráfico, hay también convenciones que se refieren a distintas tradiciones verbales ${ }^{2}$, propias de la oralidad o de la escrituralidad, respectivamente. Bajo estos diferentes aspectos, el presente artículo pretende analizar cuatro cartas de autores semicultos, es decir, personas que dominan en principio la técnica cultural de la escritura, pero cuyos textos evidencian al mismo tiempo la poca experiencia que tienen con la comunicación escrita. Las cuatro cartas son parte de una colección más amplia de cartas escritas por soldados y civiles semicultos de tres fases de la historia

1 Evidentemente, el español, como otras lenguas románicas, ha conservado grafías latinizantes que dan lugar a las excepciones del principio fonético, pero estas desviaciones no deberían considerarse como principio ya que no organizan de manera regular la relación entre sonidos y signos gráficos. Desde una perspectiva sincrónica, representan meras irregularidades (que no son por ello injustificadas).

${ }^{2}$ El término tradición verbal fue acuñado por Luis FERnANdo LARA (Lengua histórica y normatividad, 2a ed., corr. y aum., El Colegio de México, México, 2009, pp. 86-87) como traducción de Diskurstradition, término que usa la lingüista alemana Brigitte Schlieben-Lange para un conjunto de convenciones y reglas comunicativas, adquirido de forma parcialmente implícita y habitualizado por los hablantes. 
de México (el último siglo de la Colonia; el México independiente y la época de la Revolución mexicana). Este corpus de testimonios de la realidad lingüística de los estratos bajos de la sociedad se analizará en un trabajo posterior más amplio, principalmente con respecto a las tradiciones discursivas y la coherencia textual. En el presente estudio se van a examinar las particularidades gráficas, pragmáticas y discursivas de los textos, terminando con algunas observaciones acerca de las implicaciones de este análisis para la discusión ortográfica del español.

\section{EsTUdio LINGÜÍSTICO DE LA LENGUA ESCRITA}

Después de los debates a principios del siglo xx sobre la cuestión de si el estudio de la lengua escrita debería formar parte de la lingüística sensu stricto, por la presunta primacía de la lengua hablada ${ }^{3}$, una serie de autores aportan contribuciones muy valiosas al estudio de las propiedades de ambas manifestaciones de la lengua. Por un lado, se profundiza sobre las características de distintos sistemas de escritura ${ }^{4}$, en el contexto de las lenguas romances, específicamente sobre la relación entre grafemas y fonemas ${ }^{5}$. Por otro lado, se analizan las diferencias estructurales y estilísticas a nivel de texto que emanan de las circunstancias de la producción de textos escritos $\mathrm{u}$ orales $^{6}$. Una noción más clara sobre ambos aspectos se encuentra en la obra de Ludwig Söll, quien postula la disociación conceptual de

${ }^{3}$ Entre los representantes de la concepción de la lengua escrita como sistema de segundo orden están Ferdinand de Saussure, Cours de linguistique générale (1916), publicado por Charles Bally y Albert Sechehaye con la colaboración de Albert Riedlinger, Lausanne, Paris, 1979, p. 44; Leonard Bloomfield, Language, Holt, New York, 1964, p. 21, y Robert A. Hall, Introductory linguistics, Chilton, Philadelphia, 1964, p. 34, mientras que entre los autores que mantienen que la escritura, aunque evidentemente fue creada como sistema secundario, con el tiempo adquirió características propias y una cierta autonomía frente a la lengua hablada que la establece como un sistema semiótico de primer orden, destacan AARNI Penttilä \& Uuno SaArnio, "Einige grundlegende Tatsachen der Worttheorie nebst Bemerkungen über die sogenannten unvollständigen Symbole”, Erkenntnis, 4 (1934), 28-45 y 139-159; Joseph VACHek, "Zum Problem der geschriebenen Sprache”, en Selected writings in English and general linguistics, ed. Joseph Vachek, Paris, 1976, pp. 112-120, entre otros.

4 Cf., por ejemplo, Helmut Lüdtke, "Die Alphabetschrift und das Problem der Lautsegmentierung", Phonetica, 20 (1969), 147-176; y Florian Coulmas, Writing systems of the world, Oxford University Press, Oxford, 1989.

${ }^{5}$ Sobre las circunstancias de su génesis y desarrollo véase, por ejemplo, Helmut LüDtke, "Die Entstehung der romanischen Schriftsprachen”, VR, 23 (1964), 3-21.

${ }^{6}$ Cf., por ejemplo, Eugenio Coseriu, Teoría del lenguaje y lingüistica general, Gredos, Madrid, 1962, p. 320. 
medio y concepción en la producción del lenguaje. Según Söll ${ }^{7}$ se puede categorizar un enunciado o bien según el medio por el cual se transmite (código gráfico vs. código fónico) o bien según la concepción que caracteriza al texto (código escrito vs. código hablado). Obviamente, la distinción entre código gráfico y código fónico es una dicotomía que no permite grados intermedios, es decir, que un enunciado se realiza mediante signos audibles o bien mediante signos visuales, pero no por las dos vías a la vez (aunque se puede dar el caso de dos realizaciones simultáneas, desde luego, pero esto no quita que las dos sean discretas y separables). La diferenciación entre código hablado y código escrito, por otro lado, marca más bien una ubicación dentro de un continuo. Según Koch y Oesterreicher ${ }^{8}$, éste se despliega entre los polos de la inmediatez comunicativa y la distancia comunicativa que pueden ser vistos como el resultado de las diferentes situaciones comunicativas en las cuales suelen producirse los textos orales o escritos, respectivamente. Los autores hacen hincapié en que la alternación mediática representa una dimensión de la variación que no es enteramente congruente con la dimensión diafásica, lo que sustentan con la observación de que un estilo familiar en el ámbito escrito corresponde al estilo neutral en el ámbito hablado (p. 16). Más bien, tal dimensión diamésica se colocaría de manera transversal sobre las demás dimensiones de la variación lingüística, a saber, la diatópica, la diafásica y la diastrática, que según Coseriu ${ }^{9}$ constituyen el diasistema de la lengua ${ }^{10}$. De ahí que de ninguna manera se deben igualar la escrituralidad conceptual con el medio gráfico o la oralidad conceptual con el medio fónico, respectivamente. Basta con señalar que un texto que por su concepción pertenece al ámbito del código hablado puede realizarse en un medio gráfico (por ejemplo en diálogos escritos para obras de teatro, creados precisamente

7 Gesprochenes und geschriebenes Französisch, E. Schmidt, Berlin, 1974, pp. 11-19.

8 Peter Koch \& Wulf Oesterreicher, "Sprache der Nähe-Sprache der Distanz. Mündlichkeit und Schriftlichkeit im Spannungsfeld von Sprachtheorie und Sprachgeschichte”, RJ, 35 (1985), 19-24.

9 La geografía lingüistica, Universidad de la República, Montevideo, 1956.

10 Obviamente, no todos los lingüistas siguen esta interpretación. JOHANNEs КАвАтек ("Wenn Einzelsprachen verschriftet werden, ändern sie sich'. Gedanken zum Thema Mündlichkeit und Schriftlichkeit”, en Soziolinguistik und Sprachgeschichte: Querverbindungen, eds. G. Berkenbusch \& C. Bierbach, Niemeyer, Tübingen, 1994, p. 184) señala que en el momento de componer un texto (tanto escrito como oral), el hablante/escribiente hace una selección de los elementos que la lengua le ofrece por lo cual indica que la escritura y la oralidad son categorías del habla y no de la lengua. Con ello, este autor efectivamente se opone a la perspectiva que concibe la dicotomía oralidad y escritura como una categoría de la lengua aparte y aboga más bien por subsumir esta dicotomía bajo la ya conocida variación diafásica. En fin, la cuestión parece ser de perspectiva, según la cual se conceptualiza la lengua como

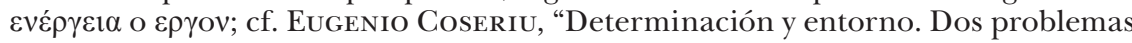
de una lingüística del hablar”, RJ, 7 (1955-56), 29-54. 
con el objetivo de imitar la inmediatez comunicativa y así de lograr autenticidad), y al revés (por ejemplo, cuando un conferencista lee en voz alta un manuscrito para un discurso público, lo que suele ser un poco fatigante).

Sea como sea, no se puede negar que los textos escritos y los hablados tienen cada uno sus propias tradiciones establecidas y que en gran parte resultan de las ya mencionadas particularidades de la situación en que se producen, y no en menor medida de las distintas circunstancias de su recepción. Así que las propiedades típicas de los textos hablados emanan del hecho de que el hablante y el oyente generalmente participan de la misma situación extra-lingüística y se puede hacer uso de gestos y mímica para acompañar y apoyar el discurso. De ahí, por ejemplo, que las expresiones deícticas suelen abundar al menos en conversaciones sobre temas cotidianos. Además, el tiempo de realización del lenguaje hablado suele ser corto, lo que ocasiona que la complejidad sintáctica y variabilidad lexical del discurso queden reducidas ${ }^{11}$. Al redactar un texto escrito, el escribiente tiene que describir la situación a un lector que está distante en el tiempo y en el espacio. Gestos y mímica sólo se pueden parafrasear por medio de palabras. Además, redactar un texto escrito generalmente tiene la posibilidad de planear y estructurar el texto con calma, pudiendo corregir y reorganizar sin dejar huellas. Por consiguiente, textos escritos tienden a emplear un lenguaje más variable y de mayor densidad lexical. La argumentación suele ser más concisa y menos redundante. Esto no es solamente resultado de las circunstancias propias del proceso de redacción, sino también una consecuencia del proceso de comunicación en el medio gráfico en general, que incluye al lector, quien tiene la posibilidad de volver a leer las partes que le resultaron incomprensibles.

\section{ANÁlisis de las CARTAS DE SOLdADOS DE LA REVOLUCión MEXICANA}

Las mencionadas particularidades de la lengua hablada y escrita se aplican en términos generales, pero sólo marcan tendencias en un continuo. Además, las tradiciones discursivas de ambos medios pueden cambiar con el tiempo y no representan sistemas homogéneos para todos los miembros de una comunidad lingüística. Es preciso mencionar en este contexto que mucho más que la lengua hablada, que forma parte de la vida cotidiana en todos los ámbitos sociales,

11 Michael A. K. Halliday (Spoken and written language, Oxford University Press, Oxford, 1985, pp. 61 ss.) especifica que la complejidad reducida del discurso hablado concierne a la densidad menor de elementos lexicales. Por el contrario, según Halliday, la lengua hablada exhibe una gramática más intrincada (p. 63). 
la familiaridad con el mundo de las letras depende de factores socioculturales y del acceso a libros y otras ocurrencias de palabras escritas. Mientras que las normas del funcionamiento de la lengua oral se aprenden de forma natural, las convenciones discursivas de la lengua escrita se aprenden en contextos más formales de aprendizaje. El dominio de tales convenciones, por ende, depende de cierta habitualidad y familiaridad con el medio gráfico por parte de los escribientes.

Los autores de los textos que se analizarán a continuación (véase Anexo) seguramente no tenían mucho acceso a la lengua escrita ni mucho menester de emplearla en su quehacer diario. Eran las circunstancias de la Revolución mexicana, y ciertas necesidades comunicativas específicas y únicas, las que motivaron a los soldados de las fuerzas revolucionarias a dirigir los presentes informes o peticiones a su superior, el general Genovevo de la $\mathrm{O}$, prominente líder militar del Ejército Libertador del Sur y compañero de Emiliano Zapata. El estudio de estas cartas es sumamente revelador en varios aspectos lingüísticos: permite acercarse a la realidad comunicativa de aquella época desde distintas perspectivas. Para los escribientes, inexpertos en esta tarea, componer un texto de impronta formal y de cierta extensión constituyó indudablemente un reto considerable. Para dar una idea de la magnitud del problema, se analizarán los textos desde tres puntos de vista diferentes que ejemplifican cada uno un aspecto particular del proceso comunicativo. El primer ángulo concierne a la simple transmisión del mensaje al medio gráfico, es decir, las dificultades que encuentran los escribientes al fijar sus ideas con signos visuales. El segundo planteamiento indaga sobre un criterio pragmático, esto es la cortesía verbal y de qué manera ésta es empleada para apoyar el objetivo comunicativo del mensaje. Finalmente, como plano lingüístico superior se analizará la estructuración textual. A este respecto, se examinará la organización temática de las cartas, o más específicamente, con qué recursos textuales se señala el cambio de tema. De esta manera, se podrán hacer afirmaciones sobre los tres diferentes aspectos del texto: el medio, la función y la forma ${ }^{12}$ y, más relevante aún, sobre cómo estos diferentes aspectos están interrelacionados.

\subsection{Ortografias personales}

Tan sólo en cuestión de ortografía habría una serie de particularidades dignas de mencionar. Por el espacio reducido del presente artículo me limitaré a señalar unas pocas generalidades que caracterizan las grafías de los textos y que anticipan los posibles temas a

12 Cf. ibid., p. 78. 
profundizar. Quizás la primera particularidad de la que se percata uno al leer las cartas es la falta de estructuración gráfica por signos de puntuación. En la carta 1 no se encuentra literalmente ningún signo de puntuación; en la carta 2 hay una sola separación de dos frases por un punto: "ora de la siembra que si ud permite que se habran terrenos nuebos hono que sino mas los salares ud manda desir. yo habri un pedasito nuebo ud medise silo sipuedo sembrarlo hono". En la carta 3 se halla una serie de comas que, no obstante, casi nunca separan frases o ideas, sino que parecen estar puestas al azar $^{13}$, incluso dividiendo sintagmas nominales, cuando se separa por ejemplo, el nombre de su complemento ("Jefe de las foerzas, del Estacamento"), el adjetivo del sustantivo ("alojamiento de ningona, clase"), o la preposición del nombre ("no les pido en, nada"). El único caso, donde un coma coincide con una pausa en el texto, es cuando se utiliza para delimitar el vocativo en la carta 3 ("Jefe de las foerzas, del Estacamento de huitzilac, tengo la honra..."). Antes de comentar esta escasez de estructuración gráfica por signos de puntuación en los textos, habría que preguntarse con qué finalidad se utiliza generalmente la puntuación, que a fin de cuentas tampoco es necesaria en la lengua hablada. Sin embargo, en la lengua hablada hacemos uso de la prosodia para transmitir contrastes sistémicos de significación ${ }^{14}$, por ejemplo, para marcar la diferencia entre una frase afirmativa y una pregunta, pero también simplemente para señalar límites entre ideas u oraciones. La variación del tono en el transcurso de la oración o de una serie de oraciones transmite, por ende, información relevante sobre la significación y el sentido comunicativos de las mismas. La puntuación en la lengua escrita se emplea en gran parte para transmitir precisamente este componente del mensaje. Facilita la clasificación y organización mental en el momento de procesar el texto. Tómense, por ejemplo, las siguientes oraciones: “...y nonca los evisto pelear para mi digo que no eran cumtricantes es cuanto lu pongo en cunosimiento Para so gobierno y Fines consiguientes" (carta 4, 11. 48-52). A primera vista, la lectura induce a pensar que los dos soldados nunca pelearon para el autor, y sólo una relectura del pasaje pone en claro que éste no los ha visto pelear entre sí. En el mismo tenor, la falta de puntuación dificulta la secuencia: “...nomas por la vorrachera andan asiendo lo Que quieren sin tener coronel adonde quiera que hentran dicen que no mas va uno de heyos como teniente coronel del coronel Crus..." (carta 1, 11. 20-26). Una primera lectura podría llevar a agrupar equivocadamente "sin tener coronel adonde quiera", interpretando que no se encuentra ningún coronel por doquier. Obviamente, al darse cuenta de que su hipótesis es

13 Se excluyen de esta observación las comas que aparentemente se utilizan para señalar la abreviación de Usted ("Ud”).

${ }^{14}$ Cf. Halliday, op. cit., p. 30. 
equivocada, el lector la reformulará mentalmente, pero sin duda la falta de señales visuales de organización dificulta la asimilación del mensaje. Resumiendo, se puede constatar que en las presentes cartas se hace una transcripción de la cadena hablada sin compensar la notransmisión de la prosodia, lo que pone en riesgo la inteligibilidad del texto por la falta de estructuración visual.

Esta observación coincide con otras dificultades evidentes, que conciernen a la separación de palabras. Como es sabido, la separación de palabras es una convención que no formó parte de la escritura alfabética durante muchos siglos, aún después de su invención. Este hecho ya indica que se requiere un esfuerzo mental considerable para identificar una forma libre mínima ${ }^{15}$. En los textos hay casos de unión de palabras que convencionalmente aparecen separadas (carta 1: nomas [no más], haver [a ver], nies [ni es], andado [han dado]; carta 2: poreso [por eso], enlos [en los], aeso [a eso], aste [ha de], delamanta [de la manta], hono [o no], medise [me dice], silo [si lo], sipuedo [si puedo], abersi [a ver si], ade [ha de]; carta 3: loque [lo que], leniega [le niega]; carta 4: mimando [mi mando], eneste [en este], emi [en mi], antenido [han tenido], seregresa [se regresa], lacaravina [la carabina], cunel [con él], llase [ya se], ecunosido [he conocido], cuntudos [con todos], evisto [he visto], medira [me dirá], enel [en él]). Es notable que se trata sin excepción de palabras gramaticales o de una combinación de un gramema y un lexema, pero nunca de una combinación de dos lexemas. Parece indicar que en la representación cognitiva de los hablantes, un lexema tiene otro estatus como palabra que un gramema. Este punto sería digno de ser tratado aparte y con una base de datos más amplia. Aquí me limito a decir que nuevamente se muestra la estrecha orientación de los escribientes hacia la lengua hablada. Ésta, en contraste con las escrituras alfabéticas, representa un continuo fónico de sílabas agrupadas en secuencias rítmicas de varias palabras. De forma que en el medio fónico no existe un análogo de los espacios entre las palabras, convencionalmente usados en el medio gráfico. Para nuestros autores esta divergencia entre los dos medios dificulta nuevamente la tarea de expresarse por medio de signos visuales.

Aparte de las dificultades mencionadas, la propia representación gráfica de las palabras constituye otro problema fundamental para los escribientes. El sistema de escritura del español, como todos los sistemas alfabéticos, se basa en el principio fundamental de segmentar la cadena sonora en sus fonemas. Sin embargo, el así llamado principio fonético nunca fue realizado cien por cien en la ortografía española, impedido desde un principio por el hecho de que se hubie-

15 En el presente artículo me abstengo de la discusión engorrosa sobre lo que constituye una palabra y, para mayor conveniencia, me oriento por las convenciones de la ortografía española. 
ra adoptado el inventario de letras del latín para representar una lengua con un sistema fonológico ya alterado. Aún así, en la época alfonsí, las convenciones ortográficas del español se caracterizaban por una alta proximidad entre la imagen sonora de las palabras y su representación gráfica ${ }^{16}$. A pesar de que con los cambios fonéticos subsiguientes aumentara la distancia entre el código fónico y el gráfico, en comparación con el francés o el inglés la ortografía española ha mantenido una relación muy estrecha con la forma oral de la lengua ${ }^{17}$. En términos de Geoffrey Sampson ${ }^{18}$, el español se puede caracterizar como un sistema plano por su orientación en la superficie fonética. Únicamente algunos rasgos etimologizantes, que hoy no son funcionales, ya que en su mayoría no se refieren a elementos morfológicos o lexemáticos ${ }^{19}$, le dan cierta profundidad diacrónica. En perspectiva sincrónica, estos rasgos etimologizantes constituyen meramente irregularidades que pueden causar dificultades a un escribiente que se orienta sólo en la pronunciación sin estar familiarizado con la Gestalt ${ }^{20}$ visual de las palabras. Por el carácter limitado de este artículo, no se puede pormenorizar en las particularidades de cada una de las grafías personales, pero sí es importante señalar algunos patrones recurrentes que ejemplifican problemas generales. En primer lugar, se pueden mencionar los problemas que causan las grafías etimologizantes, que conciernen a las alternancias entre $\langle\mathrm{b}-\mathrm{v}\rangle$ y entre $<\mathrm{j}-\mathrm{g}>$ ante $<\mathrm{i}, \mathrm{e}>$.

Evidentemente, la orientación en la pronunciación no representa ningún auxilio para la decisión por una de las alternativas, tratándose de una diferencia exclusivamente gráfica. De allí que una estrategia alfabética -es decir, una transcripción sonido por sonido- está predestinada a fracasar. Esto es ilustrado por el hecho de que en $42.3 \%$ de los casos los autores no aciertan en la elección correcta de $<$ b $>$ o

16 Cf. José Jesús de Bustos Tovar, "Spanisch: Graphetik und Graphemik. Grafética y grafémica”, en Lexikon der Romanistischen linguistik, eds. M. Günter Holtus y Christian Schmitt, Niemeyer, Tübingen, 1992, Bd. 6, 1, p. 69.

17 Aún los cambios de la así llamada revolución fonética de los siglos Xv y XVI (cf. Juan Felipe García Santos, Cambio fonético y fonética acústica, Universidad, Salamanca, 2002, p. 101) son relativamente exiguos y no afectan de manera fundamental el carácter fonológico de la ortografía española. Para una sinopsis del desarrollo de ésta véase Bustos Tovar, art. cit., o Trudel Meisenburg, "Das spanische Schiftsystem", en Schrift und Schriftlichkeit, eds. H. Günther und O. Ludwig, Berlin, 1996, pp. 1437-1441.

18 Writing systems: A linguistic introduction, Stanford University Press, Stanford, 1985, p. 200.

19 Trudel Meisenburg, art. cit., p. 264.

20 En el sentido de un todo estructurado y coherente, almacenado como esquema visual en la mente (cf. Friedrich SANDER, "Experimentelle Ergebnisse der Gestaltpsychologie”, en Bericht über den 10. Kongress über Experimentelle Psychologie in Bonn, ed. Erich Becker, Fischer, Jena, 1928, pp. 23-67). 
$<\mathrm{v}>$ (71 ocurrencias, 30 divergencias $^{21}$ ) y se equivocan en $37.5 \%$ de los

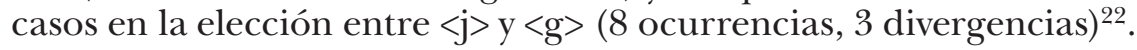
En el caso de la letra etimológica $<\mathrm{h}\rangle$, que no tiene complemento fónico en el español actual, las divergencias ortográficas muestran una tendencia muy curiosa en tres de las cartas. Mientras que en la carta 3 no se encuentra ninguna divergencia que concierna a la $<\mathrm{h}\rangle$, en las tres otras suman 43. Lo más llamativo, sin embargo, es que más que omitir la letra donde debería figurar ortográficamente (7 casos), los escribientes la insertan donde no cuadra (36 casos). Al tratarse de una letra etimológica sin complemento fónico, los autores la deben asociar como ninguna otra con la lengua escrita. La distribución entre omisiones y agregaciones erróneas sugiere la interpretación de que la $<\mathrm{h}>$ es considerada como una letra muy elegante por los autores. Esto explicaría la prevalencia de casos de ultracorrección ya que supuestamente estas $h$ contribuirían al carácter oficial y formal de la carta.

Aparte de estos problemas, que se podrían denominar panhispánicos porque atañen a todas las variedades sincrónicas del español, se notan otras dificultades específicamente latinoamericanas, resultantes de la desfonologización de $/ \mathrm{s} /: / \theta / \mathrm{y} / \mathrm{j} /: / K /$, ambas casi generales aunque no exclusivas del español de América. Para los escribientes mexicanos, la alternancia de las letras $\langle\mathrm{s}-\mathrm{c} / \mathrm{z}\rangle \mathrm{y}<\mathrm{y}-\mathrm{ll}\rangle$, por consiguiente, representa una discriminación exclusivamente gráfica no reflejada en su pronunciación. De modo que el recurso a la estrategia alfabética lleva a errores de ortografía en $37 \%$ con respecto al fonema $/ \mathrm{j} /$, representado por $<\mathrm{y}-1 \mathrm{ll}\rangle$ (27 ocurrencias, 10 divergencias). A primera vista, en el caso del fonema /s/ el porcentaje de grafías erróneas es más bajo (10\%: 370 ocurrencias, 38 divergencias). Esta observación coincide con los hallazgos de Raúl Ávila ${ }^{23}$, quien encuentra la frecuencia más baja en este error entre las otras ahí mencionadas en un estudio de textos escolares de niños de primaria. Sin embargo, si uno toma en cuenta exclusivamente las equivocaciones de $<$ s-c $>$ ante $<$ e, i $>$ uno se da cuenta de que en este contexto gráfico, al menos en las presentes cartas, los errores son mucho más frecuentes, con 29\% (116 ocurrencias, 34 divergencias $)^{24}$.

21 Se utiliza el término divergencia en vez de falta de ortografía porque este último implicaría que la norma ortográfica es en principio conocida por los autores de las cartas. El problema de ellos, sin embargo, es más bien que no tienen acceso a esta norma.

${ }^{22}$ No se contaron divergencias en los nombres propios.

23 “Ortografía española: estratificación social y alternativas" NRFH, 40 (1992), pp. 653-654. Del autor, también es interesante su trabajo "Un alfabeto fonológico práctico para el español: pros y contras y pros y contras...", en Scripta philologica: in honorem Juan M. Lope Blanch, coord. E. Luna Traill, UNAM, México, 1991, t. 2, pp. 7-19.

24 Ávila ("Ortografía española: estratificación social y alternativas") no distingue entre diferentes contextos gráficos de las $<\mathrm{s}>$ en cuanto a las frecuencias de los 
Se puede constatar, por ende, que el sistema de escritura del espanol, relativamente plano en comparación con el francés o el inglés, aumenta en profundidad para los hablantes seseantes y yeístas.

Aparte de estos problemas recurrentes y bien documentados también en otros estudios ${ }^{25}$, se encuentran, asimismo, divergencias de ortografía más individuales, como ilustra la siguiente corta selección del presente material: carta 2: cota (cuota, 1. 2), trajieron (1. 24), dijieron (1. 25); carta 3: foerzas (1. 3), precisu (1. 9), suy (1. 10), recibiu (1. 11), ningona (11. 15, 16); carta 3: suldados (11. 5, 15), emi (a mi, l. 7), hucho (1. 7), niguna (1. 8), sus pecha (sospecha, 1. 8), cun (con, 1. 10), cumo (1. 11), hulle (oye, 1. 14), umisidio (homicidio, 1. 24), inpece (empecé, 1. 25), moy itimos (muy íntimos, 1. 28), moerte (1. 31), cain (caen, 1. 35), se en curporaro cumigo (se incorporaron conmigo, 1. 42), cuntudos (con todos, 1. 45), etc. Esta lista incompleta de las grafías personales demuestra sobre todo la gran variabilidad que resulta de la orientación en la lengua hablada. La estrategia alfabética de los autores produce un sinnúmero de formas variadas que sin duda reflejan la gran variabilidad del habla individual o regional.

\subsection{Elementos de cortesía verbal}

Ya se mencionó el hecho de que el proceso de redactar un texto escrito es distinto en varios aspectos fundamentales de la producción de un texto hablado y que esto generalmente resulta en diferentes características estructurales. Aparte de estos atributos de los textos escritos, que en primer lugar son la consecuencia de las particularidades temporales y espaciales que los distinguen del lenguaje hablado, también hay diferentes tradiciones de cortesía verbal propias de ciertos textos, como las cartas. Esto concierne a distintas formas y fórmulas de tratamiento, lo mismo que a convenciones de apertura y cierre del texto, como saludos y palabras de despedida. Naturalmente, estas convenciones dependen del grado de familiaridad entre los participantes en la comunicación, y de las relaciones jerárquicas que existen entre ellos. En el caso de las cartas de soldados de la Revolución, que escriben al general Genovevo de la $\mathrm{O}$, se trata de una constelación asimétrica en cuanto a las jerarquías sociales, es decir, el general es de un rango superior en términos militares. Por la clase de asuntos que se tratan -son en primer lugar peticiones de intervenir o de mandar órdenes- se puede esperar un tono respetuoso por

errores, por lo cual no se puede hacer una declaración aquí al respecto. Sin embargo, los resultados del presente estudio sugieren que es relevante hacer esta distinción.

25 Por ejemplo, RAúl Ávila, “Ortografía española: estratificación social y alternativas". 
parte de los solicitantes. De hecho, se percibe una clara conciencia en los autores de la necesidad de expresar verbalmente el respeto y de atenuar la intrusión en la esfera del comandante en jefe. Se nota sobre todo en la cantidad de actos de habla que no contribuyen directamente al propósito general de la carta o macroacto de habla ${ }^{26}$, sino que sirven exclusivamente como saludos, declaraciones de buenas intenciones y justificaciones. Esto se debe a que una petición de un favor puede constituir de por sí un acto de impertinencia, más aún, si se trata de una petición a un superior. En términos de Brown y Levinson ${ }^{27}$, se podría hablar de un acto amenazador de la imagen negativa (negative face) del otro $^{28}$. Por este motivo, sobre todo en la carta 1 y 3 abundan las fórmulas de cortesía verbal, por medio de las cuales los autores tratan de apelar tanto a la imagen positiva como negativa del destinatario. Esto se manifiesta por ejemplo en las fórmulas de tratamiento reverenciales (carta 1: "Muy Estimado y Apresiable Sr Don Ginovevo de la O", "su merced", carta 3: "Sr jinobebo de la $\mathrm{O}$ muy Sr. mio Jefe de las foerzas, del Estacamento de huitzilac"), que reflejan simultáneamente el respeto y la distancia social entre los interlocutores.

Es bastante obvio que los escribientes no dominan plenamente estas fórmulas y convenciones de cortesía verbal de la comunicación escrita ya que no las emplean de forma eficiente y ni siquiera congruente con el propósito. Así, por ejemplo, la carta 3 se abre con una serie de fórmulas desarticuladas:

Sr jinobebo de la O muy Sr. mio Jefe de las foerzas, del Estacamento de huitzilac, tengo la honra de participar à Ud, Permitame, à Ud, recibir mi suplicas en carecidamente el fabor que pido à Ud, que se sirba à Ud, aseptar y conoser mi conducta que no es cierto como se dice ni como se save es muy precisu participar a $\mathrm{Ud}$, loque es cierto no suy tirano con ninguna desde el Dia 20 de Enero.

Claramente, el autor procura imitar el estilo de otras cartas oficiales que ha visto, copiando expresiones fijas de éstas. Sin embargo, él no acierta aquí, ya que no es capaz de incorporarlas de manera sintácticamente correcta ni pragmáticamente adecuada, pues el asunto que tiene que participar en realidad no es motivo de honra, como él afirma (1. 4). Aparte, las cartas 1 y 3 se destacan por un uso excesivo

26 Cf. Teun van Dijk, Texto y contexto. Semántica y pragmática del discurso, trad. J. D. Moyano, Cátedra, Madrid, 1980, pp. 332-339.

27 Penelope Brown \& Stephen C. Levinson, Politeness: Some universals in language usage, Cambridge University Press, Cambridge, 1978, pp. 61-62.

28 En la teoría de BRown y Levinson (id.), la imagen positiva se refiere al deseo fundamental de ser apreciado, apoyado y aceptado mientras que la imagen negativa corresponde a la exigencia de tener libertad de movimiento y de actuar sin impedimentos de otras personas. 
de la forma respetuosa del pronombre de la segunda persona (usted, su merced). Por lo que parece, los escribientes dudan del potencial de cortesía de las formas verbales del paradigma correspondiente a usted, que intrínsecamente ya expresan la forma de tratamiento, por lo cual siempre colocan la respectiva forma pronominal (por ejemplo, carta 1, 11. 9-16: “...despues de saludar á su merced Sr Don Ginovevo de la O le suplico á su merced que me haga Ud un favor de que si puede Ud pasar a Ahuatepec para que haver si puede Ud harreglar unos muchachos..."). Estos usos se podrían clasificar como casos de ultracorrección pragmática.

Las cartas 2 y 4, por el contrario, se caracterizan más bien por la escasez de fórmulas de cortesía que tradicionalmente se encuentran en las cartas formales. Al comienzo de la carta 2, por ejemplo, no se ofrece ninguna expresión formal de saludo ni de introducción aparte de la mención del destinatario sin adjetivos, tal como uno esperaría en este tipo de comunicados (carta 2: "Al $\mathrm{S}$ Coronel en jefe Ginobebo de la O Solo le comunico a ud que ya de la cota que ud himpuso yano se junta nada..."). Generalmente, se supone que el comportamiento verbal cortés forma parte de la competencia comunicativa que los hablantes nativos de una lengua adquieren de forma natural e implícita durante su socialización. Sin embargo, la comunicación por escrito se distingue de la conversación oral respecto a las tradiciones verbales, y estas usanzas se transmiten de manera distinta, es decir, se enseñan (o no) más explícitamente. Son rituales de orden social que representan un marco bastante fijo de actuación para la comunicación escrita, aún más en esta época ${ }^{29}$. La impresión que dan las presentes cartas es que sus autores sí tienen una idea de que la ocasión de redactar una carta formal requiere de un registro particular. Sin embargo, no tienen un concepto claro de los rituales verbales adecuados para esta ocasión. Ante la duda, algunos optan por un verdadero "fuego de barrera" de fórmulas de cortesía que se hallan fuera de contexto (cf. cartas 1 y 3), mientras que otros (cf. cartas 2 y 4) simplemente se abstienen de suavizar el mensaje con actos de habla que apelen a la imagen del recipiente. En ambos casos, se puede constatar una carencia de familiaridad con los rituales sociales del ámbito de la lengua escrita a la que los autores indudablemente aspiran, pero que no logran. El tipo de texto, "carta formal a un superior", sigue por lo general ciertos patrones que ordenan la secuencia de los actos de habla. Es decir, como tipos de texto tienen una particular forma prototípica que si bien no es determinista para el caso individual, sí representa modelos generales con normas y usanzas

29 Hoy en día, con el auge de nuevas formas de comunicación escrita a través de medios digitales parece que estas premisas ya no tienen la misma validez. 
más o menos fijas ${ }^{30}$. La preterición de estas normas resulta, en el caso de las presentes cartas, en un tono un tanto brusco (cartas 2, 4), o bien en perjuicio de la coherencia textual (cartas 1, 3), por la redundancia y falta de interconexión de las fórmulas de cortesía.

\subsection{Organización temática}

Podemos suponer que un objetivo de los autores al redactar las presentes cartas es lograr la coherencia de sus textos, es decir, la articulación de las cláusulas individuales para formar un conjunto razonable y entendible. Sin embargo, esta meta representa un reto singular para nuestros escribientes inexpertos, porque generalmente en la escuela no se enseña de qué manera se puede alcanzar tal conexión textual. La coherencia es especialmente amenazada cuando el autor quiere pasar de un tema a otro o de un aspecto a otro del mismo tema, puesto que en este momento hay que encontrar la solución para la transición de un asunto al siguiente. En el presente análisis, me limitaré a indagar la coherencia en ese aspecto por su valor informativo sobre la textualidad de las cartas. El obstáculo inicial lo representa el pasaje del saludo formal al primer asunto de la comunicación. En la carta 1 encontramos una apertura bastante apropiada por medio de un saludo, que incluso se separa visualmente del párrafo siguiente. No obstante, después de esta expresión formulaica, el autor muestra inseguridad al exponer el propósito de la carta, probablemente por reservas a pedirle un favor al general, por lo cual reanuda el siguiente párrafo con la partícula pues (1. 8), seguida por una repetición indirecta y redundante del saludo ("Pues hen la presente despues de saludar á su merced Sr Don Ginovevo de la O le suplico..."). El uso de pues en esta función meramente fática es claramente propio del lenguaje hablado, en el que puede ser empleado para estructurar el discurso, por ejemplo, para señalar el deseo de tomar el turno. En correspondencia, Iraset Páez Urdaneta ${ }^{31}$ describe el cambio de la palabra pues de una conjunción causal a una partícula enfática en el discurso hablado, lo que percibe como un proceso de degramaticalización ${ }^{32}$. Puesto que en el presente caso el autor de la carta no quiere expresar una relación causal, y dada

30 Cf. Wulf Oesterreicher, “Zur Fundierung von Diskurstraditionen”, en Gattungen mittelalterlicher Schriftlichkeit, eds. B. Frank, T. Haye \& D. Tophinke, Niemeyer, Tübingen, 1997, p. 29.

31 “Conversational pues in Spanish: A process of degrammaticalization?”, en Papers from the 5th International Conference on Historical Linguistics / Referate von der 5. Internationalen Konferenz für Historische Sprachwissenschaft, ed. Anders Ahlquist, Amsterdam, 1982, pp. 332 ss.

32 De manera similar, Hortensia Martínez García, "Del pues «temporal» al «causal»y «continuativo»", en Actas del congreso de la Sociedad Española de Lingüistica 
también la independencia sintáctica de pues en esta cláusula, es justificable interpretar la función de pues en esta ocasión como la de un marcador discursivo ${ }^{33}$. En contraste con el discurso hablado, en el que el uso del pues fático puede ser funcional para la organización del texto; el empleo de la partícula en este sentido en un texto escrito parece inadecuado para establecer la conexión entre las distintas partes del texto. Después, sigue la exposición del problema que es el motivo de la carta -dos soldados que molestan y extorsionan a los vecinos de Ahuatepec. Los diferentes aspectos de este problema se introducen de manera más o menos explícita como tales. Dos veces el autor emplea "no más" para iniciar una nueva cláusula (1. 21: "nomas por la vorrachera andan"; 1. 29: "no mas por el Tescal se hestan”); expresión asimismo del ámbito de la lengua oral e insólita para la estructuración de la información en el presente contexto formal. Después de la exposición del problema, el autor quiere continuar con el siguiente acto de habla, que es volver a pedir el apoyo del general. Introduce este cambio de tema con las palabras "y haura..." (y ahora), lo que pone de manifiesto el arraigo del texto en el aquí y ahora, es decir, la inmediatez comunicativa del mismo, colocándolo una vez más en el ámbito oral, a pesar de su realización gráfica. Lo mismo vale para las introducciones de los actos de habla subsiguientes, que efectivamente son meras repeticiones de actos de habla anteriores (11. 36-37: "y haver si me hase Ud ese favor..."; 1. 39: "pero lla le digo"; 1. 43: "Y hasi hes que le suplico..."). El modo repetitivo de los actos de habla del texto es otra faceta que contribuye a la impronta oral del mismo, ya que por su cualidad efímera, la lengua hablada se caracteriza generalmente por una mayor redundancia, innecesaria en los textos gráficos por su carácter permanente.

Los mismos rasgos de la oralidad se encuentran también en la carta 2, que empieza igualmente con la exposición de un problema -el retraso en el pago de las cuotas de algunos vecinos del pueblo. El paso en el texto al siguiente tema se hace de manera casi idéntica a la carta 1: "y hora quiero que ud mande" (1. 11). De carácter aún más oral son las siguientes dos introducciones de nuevos asuntos que el autor quiere comentar: "a y so delamanta" (1. 12); "ora de la siembra” (1. 14). Por su estilo casi deíctico, son buenos ejemplos de la inmediatez comunicativa que caracteriza la carta.

La carta 3 se distingue sobre todo por el uso excesivo de las expresiones formulaicas que se extiende hasta la línea 10, antes de

(Tenerife, 2-6 de abril de 1990), 1990, t. 2, p. 599, distingue dos clases de pues: el causal explicativo-continuativo y el fático.

33 Según Deborah Schiffrin, marcadores discursivos son "sequentially dependent elements that bracket units of talk..., i.e. nonobligatory utterance-initial items that function in relation to ongoing talk and text" ("Discourse markers: Language, meaning and context", en The Handbook of discourse analysis, eds. D. Schiffrin, D. Tannen \& H. E. Hamilton, Blackwell, Oxford, 2001, p. 57). 
siquiera llegar al asunto. Aparte del desequilibrio pragmático, ya comentado en el capítulo anterior, la encadenación de fórmulas incongruentes en la apertura de la carta (11. 2-10) también perjudica la coherencia del texto, puesto que no son utilizadas adecuadamente ni corresponden al propósito. En la última carta, destaca el uso reiterado e invariado de la conjunción y para unir las distintas partes de los sucesos -el homicidio por inadvertencia de un soldado por un camarada- lo que es típico del lenguaje hablado. Otro caso frecuente en todas las cartas es el de un cambio de tema sin transición textual ninguna. Desde luego, no siempre es necesario realizar una transición temática a través de medios cohesivos explícitos para mantener la coherencia del texto. Sin embargo, la falta de puntuación, ya comentada en el apartado 2.1, dificulta también la asimilación de la estructura temática del texto, como muestra el siguiente ejemplo de la carta 4: "le toca una vala a pablo y el difunto llase le dio se poltora” (1l. 39-40). En combinación con los problemas de ortografía, una primera lectura podría llegar a la siguiente interpretación: "Le toca una bala a Pablo y el difunto yace. Le dio sepultura" aunque probablemente el autor quiso decir: "Le toca una bala a Pablo y él fallece. Ya se le dio sepultura".

Resumiendo, para el cambio de tema se pueden identificar soluciones que se extienden desde nada (el cambio de tema simplemente no se advierte por medios textuales ni visuales) al empleo de marcadores discursivos claramente pertenecientes al ámbito de la oralidad o a expresiones formulaicas de un estilo altamente formal, aunque a veces empleadas inadecuadamente. La alternancia entre estos diferentes recursos estilísticos es frecuentemente brusca y refleja la poca familiaridad que los autores tienen con las tradiciones textuales del lenguaje escrito.

\section{Conclusiones}

El análisis de las cartas de escribientes inexpertos mexicanos de la época de la Revolución muestra en todos los aspectos de los textos -el ortográfico, el pragmático y el textual- las mismas tendencias. Por un lado, se manifiesta la orientación hacia el lenguaje hablado, pero, por otro, se observan intentos claros de emular las tradiciones de la lengua escrita. En otras palabras, los autores aspiran a un cierto modelo tanto ortográfico como estilístico y retórico, pero por su poca familiaridad con la lengua escrita, estas aspiraciones se ven frustradas. Precisamente por este motivo, se nota una suerte de oscilación entre la distancia y la inmediatez comunicativas. Tal variación interna en un mismo texto entre estos polos no es de por sí algo inusual, pero sí llama la atención la amplitud de la oscilación. Además, es notable 
que la inseguridad respecto a las reglas ortográficas o las formas de cortesía adecuadas puede afectar la coherencia del texto por su falta de estructuración visual y de ordenación de los actos de habla. Claramente, los problemas con la lengua escrita no se limitan a la ortografía, sino que se presentan igualmente en los demás niveles de la lengua. Así se mostró respecto al nivel pragmático y textual, pero también se puede corroborar para el léxico y la sintaxis. En suma, la lengua escrita en sus diferentes facetas forma un conjunto de niveles entrelazados. La competencia en la escrituralidad, en efecto, consiste en algo más que competencia ortográfica. Implica también conocimientos sobre las tradiciones verbales propias de la comunicación escrita, así como un dominio de la organización textual. En ambas esferas, los autores exhiben idéntica inseguridad que en el ámbito de la ortografía, como también las mismas estrategias para esquivar los problemas, es decir, una orientación hacia la lengua hablada. Claramente, la lengua escrita en su totalidad es un arca cerrada para los autores. Por el carácter holístico del ámbito de la escritura también parecen dudosas las propuestas de llevar a cabo una reforma fonocéntrica con el objetivo de alfabetizar a los grandes contingentes iletrados de las sociedades latinoamericanas, como propusieron Mateo Alemán, Andrés Bello, Domingo Faustino Sarmiento, y, en fechas más recientes, Lidia Contreras y Raúl Ávila ${ }^{34}$, por sólo nombrar algunos. Las divergencias de ortografía que encontramos en las cartas de los soldados no indican la necesidad de cambiar el sistema, sino que los escribientes no han alcanzado la etapa ortográfica, que consiste en comprender que la lengua escrita es parcialmente independiente de la hablada. El objetivo de este artículo no es denunciar errores ortográficos ni ciertos usos, sino demostrar que los escribientes aspiran a una norma que no alcanzan por falta de costumbre en ella ${ }^{35}$. Hay que enfatizar que el aprendizaje de las peculiaridades tanto mediales como pragmáticas y estructurales de los textos escritos se verifica en

${ }^{34}$ Mateo Alemán, Ortografía castellana [1609], ed. J. Rojas Garcidueñas; est. prelim. T. Navarro Tomás, El Colegio de México, México, 1950; ANDRÉs Bello y JuAN GARCía DEL Río, "Indicaciones sobre la conveniencia de simplificar la ortografía en América", Biblioteca Americana, 1823, pp. 50-66; D. F. SArmiento, Memoria (sobre ortografía americana) leída a la Facultad de Humanidades el 17 de octubre de 1843, Impr. de la Opinión, Santiago de Chile, 1843; Lidia Contreras, Ortografía y grafémica, Visor, Madrid, 1994, y RAúl Ávila, “Ortografía española: estratificación social y alternativas".

${ }^{35}$ El análisis de las cartas permite concluir que los usuarios de la lengua tienen cierta conciencia de la norma culta y las tradiciones verbales ligadas a ella. Éstas forman parte íntegra de la misma, como advierte Luis Fernando Lara: "Lejos de ser meros parásitos de la sistematicidad de las lenguas, como los sigue tratando buena parte de la lingüística moderna, los fenómenos normativos forman parte de la identidad real de las lenguas y han determinado por eso sus características internas" ("Paradigmas normativos en las lenguas históricas", Memorias de El Colegio Nacional 2008, El Colegio Nacional, México, 2009, p. 278). 
un proceso interactivo de operaciones receptivas y productivas que permite apropiarse no sólo de los aspectos estrictamente sistemáticos de representación de la lengua con signos gráficos, sino también de los aspectos tradicionales que a veces escapan a la pura lógica, pero que forman parte de la herencia cultural de una lengua histórica.

JoAchim Steffen

Universidad Nacional Autónoma de México 


\section{Carta 1}

Junio 29 De 1912

Muy Estimado y Apresia=

ble Sr Don Ginovevo de la O

donde se hencuentre Saludo á

5 su merced y á su Estado

mayor que se hencuentre Ud

de felicidad

Pues hen la

presente despues de saludar

10 á su merced Sr Don

Ginovevo de la O le suplico á

su merced que me haga Ud

un favor de que si puede

Ud pasar a Ahuatepec

15 para que haver si puede Ud

harreglar unos muchachos que

handan avosando en el pueblo pidiendo dinero y hasta amena sando la jente pasifica fin

20 que devan halgo nomas por la vorrachera andan asiendo lo Que quieren sin tener coronel adonde quiera que hentran dicen que no mas va uno de heyos

25 como teniente coronel del coronel Crus pero no dan ni he hapellido del coronel y nies sierto lo que dicen no mas por el Tescal se hestan y amas de

30 heso mas hantes handavan con el Govierno y haura le suplico a su merced que haver si puede Ud venir á desarmarlos por que llo hasta ura no

35 puedo aliviarme vien todavia no me sierran las heridas y haver si me hase Ud ese favor porque muchos andado la queja con migo pero lla le digo á Ud que

40 todavia no me halivio vien para hir á ver lo que handan hasiendo Y hasi hes que le suplico y oruego á su merced que

45 me haga Ud hese favor de venir arreglar hesos mu= chachos Es cuanto le suplica y dice $S$. $S$.

50 Antonio Baron 


\section{CARTA 2}

Al S Coronel en jefe Ginobebo de la $\mathrm{O}$

Solo le comunico a ud que ya de la cota que ud himpuso yano se junta nada y poreso mismo le mando abisar a ud ay algunos que si se prestan

5 los que tienen mas hinteres no dan menos los de mas por que hay algunos que estan al corriente y unos estan muy hatrasados y hora los que estan al corriente quie ren que asta que se igualen enlos pagos daran los de mas y hora quiero que ud mande un recado motibo aeso

10 que le mando aste desir a y so delamanta no se puede hori ta sacarla poero yo aser los mas posible de sacala tan luego como la traygan se la mando oste ora de la siembra que si ud permite que se habran terrenos nuebos hono que sino mas los salares ud manda desir.

15 yo habri un pedasito nuebo ud medise silo sipuedo sembrarlo hono y tambien le doy hasaber a ud que un endibido que esta acarrian do tabla y lamina ya estado aya en Buenavista y ademas esta rentregando los de aya y uno mas los handan biji lando los que ban a cuernavaca y le bamos aser la

20 lucha abersi lo agarramos y despues de todo le digo a ud que encargamos una cuarta de polbora yano las trajieron que ud no ade nesesitar de una poca nomas que casquiyos no ay ni balas me dijieron que ud hiba mandar es cuanto le manda

25 de $\operatorname{sir}$ S S S

Buenavistsa del Monte Mayo 21 de 1912 Manuel Ramirez

\section{CARTA 3}

Chamilpa Mayo 8 de 1912

Sr jinobebo de la $\mathrm{O}$ muy Sr. mio

Jefe de las foerzas, del Estacamento de huitzilac, tengo la honra de participar

5 à Ud, Permitame, à Ud, recibir mi suplicas en carecidamente el fabor que pido à Ud, que se sirba à Ud, aseptar y conoser mi conducta que no es cierto como se dice ni como se save es muy precisu participar a Ud, loque es cierto

10 no suy tirano con ninguna desde el Dia 20 de Enero, que recibiu de ayudante no en tenido disgustos, ni a los vecinonos de mi cargo ni llo con ellos ni ellos comigo mucho menos con à Ud, estamos unidos en este Pueblo con familas de santamaria y de

15 huitzilac no se leniega alojamiento de nin_ gona, clase y las actas de fallecimiento no les pido en, nada y si se sabe algo de min se cirva á Ud, endagar con las personas que se pueda lo que recomiendo à Ud, librarlos à Ud, y dependerlos,

20 que no aiga nobeda en el Pueblo de mi cargó protesto á Ud, mi distinguida concideracion, 
y respeto, patria y libertad, y constitucion, Francisco Osorio

\section{Carta 4}

Febrero 5 de 1914

Sr General

Genovevo de la $\mathrm{O}$

precente

5 Notifico á usted que dos suldados de mimando se enboracharon eneste Dia y llegan emi cuartel a las hucho de la noche y sin tener niguna sus pecha entre ellos de estar disgustados yo mirando

10 llegar cun gusto les dije que pasen a cenar y estos senaron a gusto charlando cumo sienpre antenido la costunbre y des pues que senaron se salieron que se hulle un tiro y entonses cuando

15 salen los de mas suldados para ver que pasava y entonses cuando lu en cuentran Zatornino avelar cun la arma de el en la mano y el hutro es Pablo espindo la estava

20 pasado de un valaso en las custillas y mirando avelar el acsidente fatal que les paso en tonses cuando seregresa en mi cuartel para darme cuenta cumo fue ese umisidio y presto que llego

25 en mi presensia inpece ecsaminarlo cumo susedio ese acsidente y me cuntes ta de esta manera que no fue echo pensado porque eran moy itimos amigos que en su casa lu asisten sienpre

30 que lle gan a lla y me cuntesta lu siguien te la moerte de Pablo susedio de este modo y van abrasados los dos cunel difonto y cumo lla estavan trastor nados ene so que ivan abrasados que se

35 cain los dos entonses cuando Dispara lacaravina y cumo al caer se chocan las armas cunel suelo entonses cuando des grasiadamente le toca una vala a pablo y el difunto llase le dio

40 se poltora.

Sr General desde el veintiocho de Diciembre se en curporaro cumigo y desde en tonses los ecunosido que eran moy vijentes y curectos y sienpre se an

45 tratado moy vie cuntudos mis mocha chos y por parte de ellos no e tenido ningon ante sedente ni tengo que aygan tenido algon enemistad a trasado 
y nonca los evisto pelear para mi

50 digo que no eran cumtricantes es cuanto lu pongo en cunosimiento Para so gobierno y Fines consiguien tes y me es plico en lo siguiente que el 55 preso lo tengo en la carcel el que a cumetio este de lito ora usted medira si le remitire enel campamento de usted espero el absolusion de usted Reforma libertad justicia y Ley

60 El coronel Marcos Perez 
